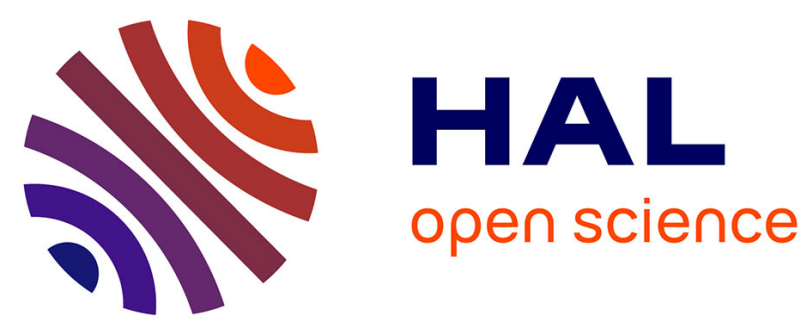

\title{
A New Approach to Tunneling
}

L. Yunpeng

\section{To cite this version:}

L. Yunpeng. A New Approach to Tunneling. Journal de Physique IV Proceedings, 1996, 06 (C5), pp.C5-49-C5-53. 10.1051/jp4:1996507 . jpa-00254386

\section{HAL Id: jpa-00254386 https://hal.science/jpa-00254386}

Submitted on 1 Jan 1996

HAL is a multi-disciplinary open access archive for the deposit and dissemination of scientific research documents, whether they are published or not. The documents may come from teaching and research institutions in France or abroad, or from public or private research centers.
L'archive ouverte pluridisciplinaire HAL, est destinée au dépôt et à la diffusion de documents scientifiques de niveau recherche, publiés ou non, émanant des établissements d'enseignement et de recherche français ou étrangers, des laboratoires publics ou privés. 
JOURNAL DE PHYSIQUE IV

Colloque C5, supplément au Journal de Physique III, Volume 6, septembre 1996

\title{
A New Approach to Tunneling
}

\author{
L. Yunpeng, L. Cunzhi and L. Enze \\ Physics Department, Xidian University, Box 271, Xi'an 710071, China
}

\begin{abstract}
A new numerical algorithm for tunneling is presented. This algorithm can be used to analyse an onedimensional potential barrier with an arbitrary profile. It is able to calculate not only the transmission coefficient accurately, but also the phases of the transmitted and reflected waves. Using the Schrodinger equation and plane waves, we introduce a tunneling matrix to relate the transmitted and incident wave functions. Thus the transmission coefficient and phase can be calculated from the tunneling matrix. To test the accuracy of this algor ithm, we calculate the transmission coefficients and phases for a double - barrier (resonant tunneling) and a triangular barrier (field emission), and make comparisons with corresponding analytical values. It shows that this algorithm is fast, accurate and efficient.
\end{abstract}

\section{INTRODUCTION}

The quantum tunnel effect is the theoretical foundation of various tunnel devices. Therefore the calculation of the tunneling current has been a subject of interest for many years. In order to calculate the tunneling current, the transmission coefficient must be calculated. Up to now the transmission coefficient has been calculated using various approximate methods, for example, the semi-classical method (WKB) [1 - 3] and the pertubation method [4]. The advantages of these methods are that they are simple in their formulae, and are able to give analytical expressions for the tumneling current in some simple cases. Their outstanding defect is that the calculated errors are large and difficult to estimate.

In this paper we present a new approach to tunneling and a corresponding algorithm for the transmission coefficient. Analysis shows that this algorithm is very accurate, and its errors can be estimated.

\section{THEORETICAL PRINCIPLES}

Figure 1 shows the potential profile of an one-dimensional barrier $V(x)$, where $m$ and $E$ are the mass and energy of incident particle, respectively. The wave function $Y(x)$ is determined from the time-independent Schrodinger equation

$$
Y^{\prime \prime}=C[V(x)-E] Y, \quad C=2 m / \hbar^{2}
$$

where the primes on $Y(x)$ indicate derivative with respect to $x$, and $\hbar$ is Planck's constant. On the left - hand side of the barrier there are incident and reflected plane waves,

$$
Y(x)=A \exp [j(P a+K x)]+B \exp [j(P b-K x)], x<=0
$$

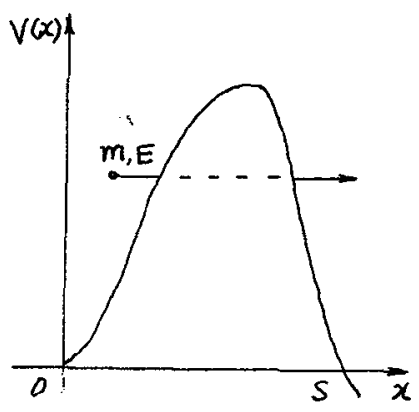

Figure 1:One--dimensional potential barrier. 
where $K=(C E)^{1 / 2} ; A, B$ and $P a, P b$ are the amplitudes and phases of the incident and reflected waves (relative to the transmitted wave), respectively. On the right-hand side of the barrier there is the transmitted wave,

$$
Y(x)=G(x), \quad x=>s
$$

where $G(x)$ is a known function determined by the boundary condition at the right - hand side of the barrier.

Under the boundary condition ( 3 ), the equation (1) can be solved by a numerical method. For example, in the Numerov algorithm [5] the difference formula corresponding to equation (1) is

$$
\begin{gathered}
\left(1-W_{i}\right) Y_{i}=\left[\left(2+10 W_{i+1}\right) Y_{i+1}+\left(W_{i+2}-1\right) Y_{i+2}\right] \\
i=N-2, N-3, \ldots, 1,0
\end{gathered}
$$

where $\mathbf{Y}_{1}=\mathbf{Y}\left(\mathbf{x}_{1}\right), \mathbf{x}_{1}=\mathbf{i} \Delta \mathbf{x}, \mathbf{s}=\mathbf{N} \Delta \mathbf{x}$

$$
W(x)=C \Delta x^{2}[V(x)-E] / 12
$$

Using (3) we have

$$
Y_{i}=G\left(x_{i}\right), i=N, N-1
$$

Thus we can get a numerical solution of $Y(x)$ in the whole region. This algorithm has the local error $O\left(\Delta x^{6}\right)$, therefore its calculated resul ts have very high accuracy.

Taking the real and the imaginary parts of the wave function, $Y(x)=Y_{1}(x)+j Y_{2}(x)$, and substituting into equations (1) - (3), we get

$$
\begin{aligned}
Y_{a}^{\prime \prime}= & C[V(x)-E] Y_{a}, \quad a=1,2 \\
Y_{1}(x)= & A \cos (P a+K x)+B \cos (P b-K x), \quad x<=0 \\
Y_{2}(x)= & A \sin (P a+K x)+B \sin (P b-K x), \quad x=>s
\end{aligned}
$$

On the other hand for a free particle with the wave vector $K$, the wave function is

$$
Z(x)=\exp (j K x)=Z_{1}(x)+j Z_{2}(x), \quad-\infty<x<\infty
$$

The corresponding equations are

$$
\mathrm{Z}_{\beta}=-C E \mathrm{Z}_{\beta}, \quad \beta=1,2
$$

Multiplying (7) by $Z_{\beta}$ and (11) by $Y_{a}$, subtracting the former from the latter, and integrating from 0 to s, we get

$$
\left[Z_{\beta} Y^{\prime}{ }_{a}-Y_{a} Z_{\beta}^{\prime}\right]_{0}^{s}=\mathrm{KM}_{\beta a}, \quad \alpha, \beta=1,2
$$

where $M$ is the tunneling matrix,

$$
M_{\beta a}=C / K \int_{0}^{s} Z_{\beta}(x) V(x) Y_{a}(x) d x,
$$


In these integrands the functions $V(x)$ and $Z_{\beta}(x)$ are known, and $Y_{\alpha}(x)$ have numerical sol utions, so these integrations can be evaluated by numerical method, for example, the Simpson quadrature with local errors $O\left(\Delta x^{5}\right)$. Substituting $(8)-(10)$ into the left-hand side of equation (12), we get four formulae,

$$
\begin{gathered}
A \sin (P a)-B \sin (P b)=M_{11}-G_{1}^{\prime}(s) \cos (K s) / K-G_{1}(s) \sin (K s) \\
A \sin (P a)+B \sin (P b)=M_{22}-G_{2}^{\prime}(s) \sin (K s) / K+G_{2}(s) \cos (K s) \\
A \cos (P a)+B \cos (P b)=M_{21}-G^{\prime}{ }_{1}(s) \sin (K s) / K+G_{1}(s) \cos (K s) \\
A \cos (P a)-B \cos (P b)=-M_{12}+G^{\prime}{ }_{2}(s) \cos (K s) / K+G_{2}(s) \sin (K s)
\end{gathered}
$$

Thus $\mathrm{A}, \mathrm{B}$ and $\mathrm{Pa}, \mathrm{Pb}$ may be solved from (14), and the reflection and the transmission coefficients are

$$
R=B^{2} / A^{2} \quad \text { and } \quad T=1-R
$$

respectively. The phases of the reflected wave and the transmitted wave ( relative to the incident wave) are

$$
P_{r}=P b-P a \quad \text { and } \quad P_{t}=-P_{a}
$$

respectively.

\section{APPLICATION TO DOUBLE-BARRIER}

For a diode with a double-barrier tunnel junction, there is resonant tunneling. Figure 2 shows the potential profile for a rectangular double - barrier. For this case, the transmitted wave is a plane wave, $G(x)=\exp (j K x)$,

$$
G_{1}(x)=\cos (K x), G_{2}(x)=\sin (K x)
$$

For this simple case, we can get an analytical formula for the transmission coefficient by solving the Schrodinger equation [9],

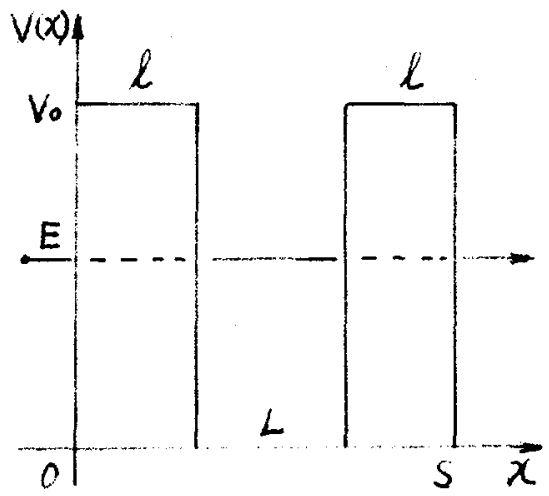

Figure 2:Rectangular double-barrier.

$$
\begin{gathered}
T o=1 /|Z o|^{2} \\
Z o=\left[\cos h(q l)+\frac{1}{2} j \alpha \sin h(q l)\right]^{2} \cdot \exp (-j 2 K L)+\frac{1}{4} \beta^{2} \sin h^{2}(q l)
\end{gathered}
$$

where $q=\left[C\left(V_{0}-E\right)\right]^{1 / 2}$, and $\alpha=q / K-K / q, \beta=q / K+K / q$. The semiclassical formula $(W K B)$ is $[7]$

$$
\begin{aligned}
& T_{2}=1 /\left|Z_{2}\right|^{2} \\
& Z_{2}=\exp (2 q l)+[\exp (2 q l)-1] \exp (j 2 K L)
\end{aligned}
$$

In calculations the values of $\mathrm{Vo}, 1$ and $L$ are set as $0.5 \mathrm{eV}, 5 \AA$ and $5 \AA$, respectively. The incident particle is an electron. Table 1 shows the calculated results, where $T_{1}$ is obtained by use of the algorithm in this paper, using $\Delta x=0.01 \AA$. It may be seen from the table that the values of $T_{1}$ are coincident with the values of To very well. The values of $T_{2}$ have large errors. 
Table 1. Transmission coefficients and phases for the rectangular double - barrier with $V_{o}=0.5 \mathrm{eV}$, and $\mathbf{l}=\mathbf{L}=5 \AA$.

\begin{tabular}{|c|c|c|c|c|c|}
\hline $\mathbf{E}(\mathrm{eV})$ & $\mathbf{T}_{\mathbf{0}}$ & $\mathbf{T}_{1}$ & $\mathbf{T}_{2}$ & $\mathbf{P t}$ & $\mathbf{P r}$ \\
\hline 0.20 & 0.04068 & 0.04008 & 0.00568 & -0.30833 & -1.58265 \\
0.25 & 0.22774 & 0.22604 & 0.01935 & -0.22806 & -1.09789 \\
0.30 & 0.99982 & 0.99970 & 0.09261 & +0.65058 & -0.03770 \\
0.35 & 0.46579 & 0.46616 & 0.64165 & +1.33637 & +1.17571 \\
0.40 & 0.26352 & 0.26326 & 0.83411 & +1.42434 & +1.57706 \\
0.45 & 0.20766 & 0.20727 & 0.63172 & +1.39218 & +1.83887 \\
\hline
\end{tabular}

\section{APPLICATION TO TRIANGULAR BARRIER}

For the field electron emission from a metal the model potential is

$$
V(x)=V_{0}-e F x, x>0
$$

where e and $F$ are the magnitude of the elementary charge and the applied el ectric field, respectively. In order to compare with the analytical values the image force is neglected. For this case the transmitted wave is an Airy function, $G(x)=\mathbf{A i}(u)-j B i(u)$, where $u=u_{0}-x / \lambda, \lambda$ is the characteristic length, $\lambda=1 /(\mathrm{CeF})^{1 / 3}$ and uo $=\mathrm{C}\left(\mathrm{Vo}_{\mathrm{o}}-\mathrm{E}\right) \lambda^{2}$.

For this simple case, we can get an analytical formula of the transmission coefficient by solving the Schrodinger equation [10],

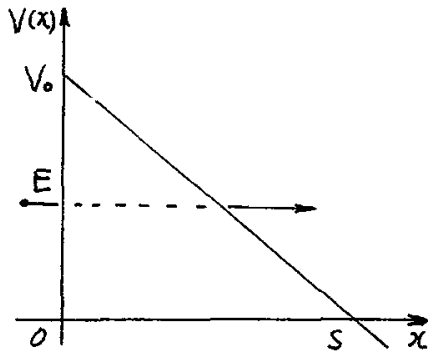

$$
T o=4 /(\pi K \lambda Z)
$$

Figure 3, T riangular barrier.

$$
\mathrm{Z}=A i^{2}+B i^{2}+\left(A i^{\prime^{2}}+B{i^{\prime}}^{2}\right) /(K \lambda)+2 / \pi K \lambda
$$

where the prime on the Airy functions $A i$ and $B i$ indicates a derivative with respect to the argument. The argument of the Airy function $\mathrm{Ai}(u)$ and $\mathrm{Bi}(u)$ is given by $u=$ uo. The semiclassical formula (WKB) is

$$
T_{2}=\exp \left(-4 / 3 u_{0}^{3 / 2}\right)
$$

Table 2. Transmission coefficients for the triangular barrier with $\mathrm{Vo}=12 \mathrm{eV}$, and $\mathrm{F}=0.5 \mathrm{~V} / \AA$.

\begin{tabular}{|c|c|c|c|}
\hline $\mathrm{E}(\mathrm{eV})$ & $\mathbf{T}_{0}$ & $\mathbf{T}_{1}$ & $\mathbf{T}_{2}$ \\
\hline 5 & $2.0344 \mathrm{E}-11$ & $2.0345 \mathrm{E}-11$ & $1.0275 \mathrm{E}-11$ \\
6 & $3.8214 \mathrm{E}-09$ & $3.8229 \mathrm{E}-09$ & $1.9063 \mathrm{E}-09$ \\
7 & $4.5839 \mathrm{E}-07$ & $4.5855 \mathrm{E}-07$ & $2.3261 \mathrm{E}-07$ \\
8 & $3.3579 \mathrm{E}-05$ & $3.3601 \mathrm{E}-05$ & $1.7928 \mathrm{E}-05$ \\
9 & $1.4005 \mathrm{E}-03$ & $1.4015 \mathrm{E}-03$ & $8.2620 \mathrm{E}-04$ \\
10 & $2.8859 \mathrm{E}-02$ & $2.8860 \mathrm{E}-02$ & $2.0983 \mathrm{E}-02$ \\
\hline
\end{tabular}


In calculations the values of $\mathrm{Vo}$ is $12 \mathrm{eV}$, and the incident particle is an electron. Tables 2 and 3 show the calculated results, where $T_{1}$ is obtained by use of the algorithm in this paper, using $\Delta x=0.01 \AA$. In table 2 the electric field is fixed, $F=0.5 \mathrm{~V} / \AA$, the electron energy $E$ is changed. In table 3 the electron energy is fixed, $E=$ $6 \mathrm{eV}$, the electric field $F$ is changed. It may be seen from the tables that the values of $T_{1}$ are coincident with the values of $T 0$ very well. The values of $T_{2}$ have large errors.

Table 3. Transmission coef ficients for the triangular barrier with $V_{0}=12 \mathrm{eV}$, and $\mathrm{E}=6 \mathrm{eV}$.

\begin{tabular}{|c|c|c|c|}
\hline $\mathbf{F}(\mathbf{V} / \AA)$ & $\mathbf{T}_{0}$ & $\mathbf{T}_{1}$ & $\mathbf{T}_{2}$ \\
\hline 0.5 & $3.8214 \mathrm{E}-09$ & $3.8226 \mathbf{E}-09$ & $1.9063 \mathbf{E}-09$ \\
0.6 & $1.0859 \mathrm{E}-07$ & $1.0862 \mathbf{E}-07$ & $5.4137 \mathbf{E}-08$ \\
0.7 & $1.1861 \mathbf{E}-06$ & $1.1862 \mathbf{E}-06$ & $5.9098 \mathrm{E}-07$ \\
0.8 & $7.1275 \mathrm{E}-06$ & $7.1290 \mathrm{E}-06$ & $3.5492 \mathbf{E}-06$ \\
0.9 & $2.8757 \mathbf{E}-05$ & $2.8761 \mathbf{E}-05$ & $1.4311 \mathbf{E}-05$ \\
1.0 & $8.7783 \mathrm{E}-05$ & $8.7800 \mathbf{E}-05$ & $4.3662 \mathbf{E}-05$ \\
\hline
\end{tabular}

\section{CONCLUSIONS}

In this paper a new approach to tunneling is presented. The corresponding algorithm can be applied to an arbitrary one-dimensional potential barrier, and the calculated results have very high accuracy. The algorithm can calculate not only the transmission coefficient, but also the phases of the reflected and transmitted waves.

\section{References}

[1] Brinkman W. F. ,et al. ,J. Appl. Phys. 41(1970)1915.

[2] Coleman R. V. ,et al. ,Phys. Rev. B29(1984) 4246.

[3] Ma Wengan, et al. ,Acta Physica Sinica (China), 40(1991) 483.

[4] Bardeen J. ,Phys. Rev. Lett. ,6(1961)57.

[5] Koonin S. E. , Computational Physics (The Benjamin/cummings Publishing Company, Inc. 2727 Sand Hill Road Menlo Park, CA 94025, 1986)Ch. 3.

[6] Inaba H. ,et al. ,J. Appl. Phys. (Japanese) , 28(1989)2201.

[7] Zohta Y. ,Phys. Rev. B 41(1990)7879.

[8] Glazer Y. and Gitterman M. ,Phys. Rev. B43(1991)1855.

[9] Merzbacher E. ,Quantum Mechanics (Wiley,New York, 1986) \$6.

[10] Jensen K.L. and Ganguly A. K. ,J. Appl. Phys. 73(1993) 4409. 